\title{
NEARBY OPEN CLUSTERS AND HR DIAGRAM CALIBRATION
}

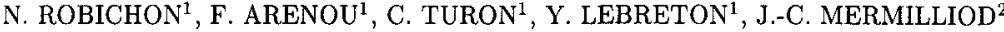 \\ 1 DASGAL - CNRS URA 335 \\ Observatore de Paris, 5, place J. JANSSEN, F92195 Meudon cedex \\ ${ }^{2}$ Institut d'astronomie, Université de Lausanne \\ CH1290 Chavannes-des-Bois
}

13 clusters have been analysed (Coma Ber, the Pleiades, IC 2602, IC 2391, Praesepe, Alpha Per, NGC 6475, Blanco 1, Stock 2, NGC 6633, IC 4756, NGC 2516 and NGC 3532). Cluster members have been selected using Hipparcos proper motions and parallaxes. The mean cluster parallaxes have been determined using Hipparcos intermediate data (abscissae on the Reference Great Circles) taking into account star to star correlations. Both selection of members and computation of cluster mean parallaxes are described in Mermilliod et al., 1997 and Robichon et al., 1997.

For each cluster, a selection of fainter stars in the 'Base des Amas' (Mermilliod, 1988), based on photometric data as well as radial velocities and proper motions when available, has been done. From this selection and the computed mean parallaxes, the location of the 13 cluster sequences in the observational HR diagram is derived very accurately, at least for the closest clusters for which good photometry and small errors on the mean parallaxes are available. Relative positions of cluster sequences cannot only be explained by differences between cluster metallicities. For example, the Pleiades sequence is 0.5 magnitude bellow the Praesepe sequence whereas the adopted metallicities (obtained spectroscopically i.e. whithout any assumption on the helium content) are respectively -0.034 and +0.10 .

Theoretical ZAMS (Lebreton, 1997) computed for each cluster according to its metallicity and a grid of Helium content has been achieved in order to estimate roughly the helium abundance of each cluster by comparison with the observed sequences. The scatter of the helium abundance $Y$ from the less abundant cluster (Praesepe: $Y \simeq 0.25$ ) to the richest one (Pleiades: $Y \simeq 0.30$ ) is about 0.05 .

These results are crucial for any further calibration of photometric parallaxes as well as for constraining theoretical models of internal structure and stellar evolution. Such a scatter in helium content is difficult to explain and is to be confirmed with more accurate cluster metallicities and $\left(T_{\text {eff }}\right.$, colour) relations. Furthermore, for the majority of the 13 clusters, accurate photometric data are lacking. In particular narrow band photometry such as Strömgren photometry would be very useful.

\section{References}

Lebreton, Y. (1997), in preparation

Mermilliod, J.-C., Turon, C., Robichon, N., Arenou, F. and Lebreton, Y. (1997), Hipparcos Venice'g7, ESA SP-402, p. 643

Mermilliod, J.C. (1988), Bull. Inf. Centre Données Stellaires, Vol. no. 35, pp. 77-91

Robichon, N., Arenou, F., Turon, C., Mermilliod, J.-C. and Lebreton, Y. (1997), Hipparcos Venice'g7, ESA SP-402, p. 567 